\title{
Selecting the correct Data Mining Method: Classification \& InDaMiTe-R
}

\author{
Prof.Thakur Ritesh ${ }^{1}$, Mr. Adsure Sharad ${ }^{2}$, Miss. Ashwini G. Sagade ${ }^{3}$ \\ ${ }^{I}$ (Associate Professor, Computer Engineering, IOKCOE,Pune University, India ) \\ ${ }^{2}$ (Computer Engineering, IOKCOE,Pune University, India) \\ ${ }_{3}^{3}$ (Computer Engineering, IOKCOE, Pune University, India)
}

\begin{abstract}
One of the most difficult tasks in the whole KDD process is to choose the right data mining technique, as the commercial software tools provide more and more possibilities together and the decision requires more and more expertise on the methodological point of view. Indeed, there are a lot of data mining techniques available for an environmental scientist wishing to discover some model from her/his data. This diversity can cause some troubles to the scientist who often have not a clear idea of what are the available methods, and moreover, use to have doubts about the most suitable method to be applied to solve a concrete domain problem. Within the data mining literature there is not a common terminology. A classification of the data mining methods would greatly simplify the understanding of the whole space of available methods. In this work, a classification of most common data mining methods is presented in a conceptual map which makes easier the selection process. Also an intelligent data mining assistant is presented. It is oriented to provide model/algorithm selection support, suggesting the user the most suitable data mining techniques for a given problem.
\end{abstract}

Keywords: Knowledge Discovery from Databases, Data Mining, Intelligent Decision Support System case Base Reasoning.

\section{INTRODUCTION}

The classical scheme of Knowledge Discovery refers the following steps to complete the high level process of KDD, very often also called simply Data Mining:

- Developing and understanding the domain, capturing relevant prior knowledge and the goals of the end-user.

- Creating the target data set by selecting a proper set of variables or data samples.

- Data cleaning and preprocessing. Quality of result strongly depends on the quality of input data, and therefore the preprocessing step is crucial (Gibert et al 2008b).

- Data reduction and projection: Depending on the problem, it may be convenient to simplify the considered set of variables. The aim here is to keep a relevant set of variables describing the system adequately and efficiently (Gibert et al 2008b).

- Choosing the data mining task, with reference to the goal of the KDD process. From clustering to time series forecasting, many different techniques exist for different purposes, or with different requirements.

- Selecting the data mining algorithm/s: once the task is decided and goals are codified, a concrete method (or set of methods) needs to be chosen for searching patterns in the data. Depending on the choice of techniques, parameter optimization may or may not be required

- Data mining: Searching for patterns in data. This will be significantly improved if previous steps were performed carefully.

- Interpreting mined patterns. This is crucial if the discovered patterns have to support effective improvement of expert's knowledge about the analyzed phenomenon or further decision-making (Gibert et al 2010, Gibert et al 2008). If results look inconsistent possible further iteration of previous steps may be required to refine the analysis.

○ Consolidating discovered knowledge: Documenting and reporting results, or using them inside the target system.

Most of the commercial Data Mining systems provide collections of several preprocessing, data mining and support-interpretation tools, which have to be properly combined by the data miner itself to build a correct KDD process for every particular application. One of the most difficult tasks is to choose the right data mining technique, as the commercial software tools provide more and more possibilities together and the decision requires more and more expertise on the methodological point of view.

In (Gibert et al 2008b) a high level description of a number of Data Mining techniques was presented in order to 
provide elements to environmental scientists to decide what to do in front a real problem. In that case I presented the techniques that I presumed could be more used for making environmental KDD, and I presented them grouped by technical proximity between them. However, in the past years I have been experiencing that either experts or data miners choose the data mining technique by using two main parameters which have nothing to do with technical characteristics of the choice. After these experiences, I strongly belief that the final choice depends basically on:

- The main goal of the problem to be solved.

- The structure of the available data.

However, providing a conceptual map of data mining techniques regarding the parameters used by human beings to decide on the right technique for a particular application, is of great help on:

- $\quad$ Modeling the decision process itself.

- Helping non-expert data miners to improve their decisions.

- $\quad$ Building technical data miner recommenders that in the future can be included at a higher level in Data Mining systems.

In this work, I present a classification of most common Data Mining techniques oriented to support the decisional problem of choosing the right one in real applications and the advantage of using it as a reference on the construction of intelligent data mining techniques recommenders (InDaMiTe-R) is discussed. In the second part of the paper a first proposal on InDaMiTe-R is presented and evaluated. Finally, conclusions and future work are discussed.

\section{Classification Of Data Mining Techniques Oriented To Decision-Making}

As I said before, I observed that main parameters taken into account by humans to choose the proper data mining technique in a real application are:

- The main goal of the problem to be solved.

- $\quad$ The structure of the available data.

According to that, I elaborated the classification displayed in Fig. 1. which includes some of the most popular data mining techniques useful for environmental scientists.

The higher level division is taking into account the basic distinction between having or not a reference variable to be explained (response variable). Left part refers to non-supervised methods, without response variable, in where the main goal is a better cognition of the target phenomenon and description is enough as a result. Whereas right part refers those supervised models oriented to re-cognition, where a response variable is to be explained and prediction is pretended.

At a second level, for methods oriented to description, the main division regards the interest of describing relationships between objects (raws of data matrix), which are labeled as descriptive methods, or describing relationships between variables (columns of data matrix), labeled as associative methods.

For methods oriented to prediction, here the main distinction regards the nature of the response variable: while discriminant methods explain or predict qualitative variables, the classical predictive methods refer to quantitative response variables.

Because of variety, discriminant models include a further level of subdivision. Rule-based reasoning methods group methods providing explicit knowledge model, which can be expressed by formal rules or not, to be applied for further prediction; in case-based reasoning methods the predictive model is implicit in historical data; the third option is a mixture between prior explicit knowledge model and iterative refinements based on future data (Bayesian learning).

Finally, in the presented conceptual map of Data Mining techniques, different colors have been used for methods coming from the field of Artificial Intelligence or Statistics, and additional information about more recent multi disciplinary proposals which can be classified in the intersection AI\&Stats is also provided. As discussed in previous works (Gibert et al 2008b, Gibert et al 2010) these hybrid techniques use to be more powerful for modeling very complex domains, as environmental systems are. 
It is presented here a very brief description of all the methods included in this classification, just providing the minimum information to make the final choice.

- Conceptual clustering: Provides grouping of homogeneous objects. Requires hypothesis about the number of classes to be found. Results are directly understandable. Usually do not work with very big data sets.

- Statistical clustering: Provides grouping of homogeneous objects. Might not require the number of classes. Can be efficient with big data sets. Sometimes difficult to understand the meaning of grouping provided.

- Clustering based on rules: Provides grouping of homogeneous objects. Do not require number of classes as input. Can introduce prior expert knowledge as semantic bias. Guarantee interpretability of results and coherence with prior expert knowledge.

- Association rules: Provides patterns of associated values of variables and frequencies of appearance.

- Model-based reasoning: Provides formal model of the causal relationships among the domain variables, by providing models for the dependencies among variables.

- Qualitative reasoning: Provides qualitative model of the causal relationships among the domain variables, by representing which variables increase or decrease values as a consequence of modifications in the values of other variables.

- Principal component analysis: Provides graphical representation to see numerical variables which behave associated or not. Extra work required to interpret results.

- Simple correspondence analysis: Provides graphical representation to see modalities of two qualitative variables which behave associated or not. Extra work required to interpret results.

- Multiple correspondence analysis: Provides graphical representation for associations among modalities of various qualitative variables. Extra work required for interpretation.

- Bayesian networks: Provides graphical interpretation of causal relationships between variables together with conditional probabilities.

- Instance-based learning: Uses historical data to classify a new instance of a problem in a predefined set of classes.

- $\quad$ Rule-based classifiers: Provide a set of classification rules that can be used later to evaluate a new case and classify in a predefined set of classes.

- Decision trees: Provide a graphical representation of a tree with conditions associated to nodes that permit to classify a new instance in a predefined set of classes. Problems with very big data sets. It works with qualitative variables.

- Discriminant analysis: Provides an algebraic discriminant function and a cut-off as the rule to decide between two groups for a new instance. Only for numerical variables, two predefined classes and works only under linear separably classes.

- Support Vector Machines (SVMs): They can provide discriminant functions to distinguish between two predefined classes that can be non-linearly separable.

- Box plot-based induction rules: Provide a set of probabilistic classification rules that can be used later to classify a new instance in a predefined set of classes.

- $\quad$ Regression-trees: Provide decision trees for prediction of numerical values. Each leaf has a numerical value, which is the average of all the training set values that the leaf, or rule, applies to.

- Model trees: Provide regression trees combined with regression equations. The leaves of these trees contain regression equations rather than single predicted values. A model tree approximates continuous functions by several linear sub models.

- Naïve Bayes classifier: Provides an adaptative classifier that can improve initial knowledge-based predictions for the class of a new instance by refining the model on the basis of the evidences provided by the whole history of processed cases.

- Connexionist models: Include all artificial neural networks models. Permit to predict the value of one or more variables for a new instance on the basis of non-linear combination of the values of several input variables and intermediary layers.

- Simple linear regression: Predicts the value of a quantitative variable for a new instance as a linear equation of a single numerical variable. Requires normality \& linearity.

- Multiple linear regression: Predicts the value of a quantitative variable for a new instance a s a linear equation of several numerical variables. Requires normality, linearity and independence

- Analysis of Variance: Predicts the value of a quantitative variable for a new instance as a linear combination of one or two qualitative variables. Requires conditional normality, linearity and independence.

- Generalized Linear Models: Predicts the value of a quantitative variable for a new instance as a linear combination of several numerical and qualitative variables. 
- Time series: Predict the value of a quantitative variable for a future instance as a linear combination of past values of the same variable.

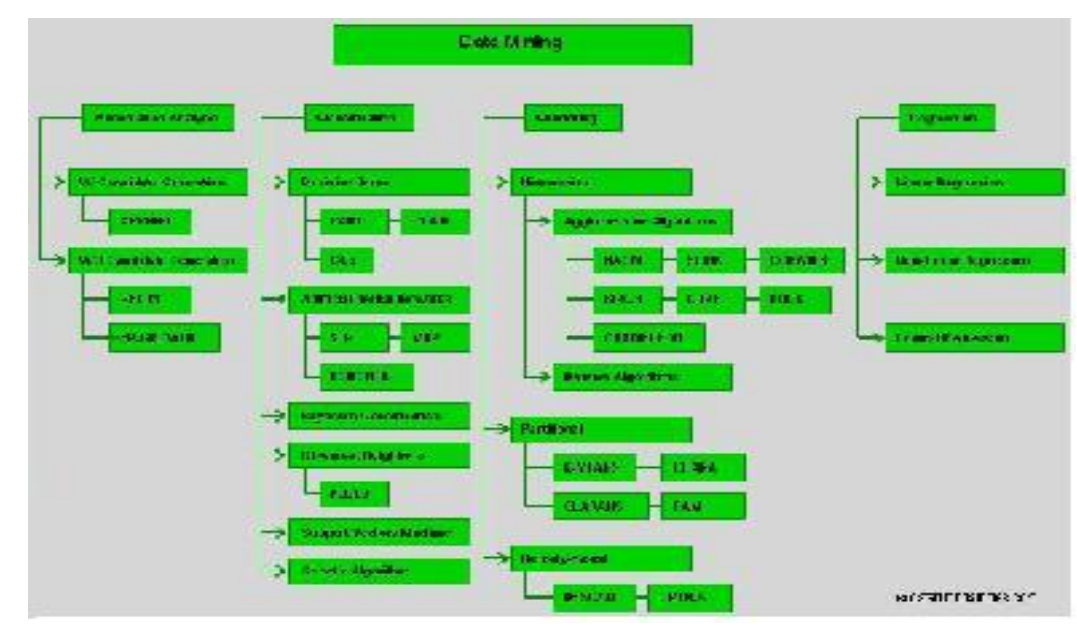

Figure 1: Classification of Data Mining Techniques

\section{An Intelligent Data Mining Recommender}

As evidenced in the previous section, there are many difficult and technical decisions that the data miner has to face in order to obtain the best outcome for a given dataset and user's goals. Selecting the machine learning or statistical method more appropriate, once a family of methods is found, deciding which training parameters are most appropriate or which particular technique is more convenient are some examples. Furthermore, most data mining commercial software tools either do not provide intelligent assistance for addressing the data mining process or tend to do so in the form of rudimentary "wizard-like" interfaces that make hard assumptions about the level of background knowledge required by a user in order to effectively use the system.

At a first sight, it seems that building some knowledge-based system including as decision- rules some translation of the conceptual map presented in previous section should be the better option for building an intelligent assistant to choose the right data mining technique to be used in a specific application.

However, it is obvious that, being that map a non-exhaustive classification of data mining techniques, but the most common ones in environmental sciences, as the tendencies or needs change in the future, new refinements of the map will be required, with the consequent modifications on the assistant. Also, the number of data mining techniques available grows incredibly fast every day and this means that the knowledge-based approach is constrained to continuous reviews and upgrades. On the other hand, the number of decisions made by an expert data miner to find the right subfamily of techniques (hierarchical clustering, or partitioned or fuzzy), the right parameters of execution (once decided hierarchical clustering, choose the algorithm, the metric, the aggregation criterion, sometimes, weight on the metrics) in a particular case is so complex that it becomes difficult to make explicit in a conceptual map.

That is the reason why I propose to move to a case-based reasoning approach, much more flexible to changes in the future on the methodological framework and using implicitly the expertise of data miners by means of past experiences. In fact, a key characteristic that any intelligent data mining assistant should possess is the capacity to learn from past user's experiences, so the system can help the user to avoid the repetition of mistakes and motivates the knowledge reuse, and on the other hand, to adapt to new possibilities by including them in the system easily. Thus, a non-expert data miner could take advantage of the experiences of others users facing similar problems [Charest et al., 2006]. And an expert can propose new solutions based on more recent trends.

\section{Case Study}

An example of use is presented for illustration. A certain data miner (Maria) wants to conduct a classification for a given dataset (D1). She wonders the better DM classification algorithm and configuration parameters for D1, and she starts the DM assistant.

First of all, Maria is asked to provide the type of task, that in this case it consists of a classification problem. After that, the system automatically extracts the most relevant metadata from D1 taking into account that the user's goal is a classification task. Some examples of metadata information that may be obtained in this case would be the number of classes, the entropy of the classes and the percent of the mode category of the class. Then, the user is asked to provide some application restriction, and in this case, the restrictions of Maria 
are that the model has to be as accurate as possible and interpretable. With all this information the system generates a recommendation consisting of two DM algorithms: the ID3 (decision tree type) and the CN2 (rule induction type). Maria executes the two proposed algorithms with the predefined configuration parameters and validates the results. As the evaluation of the ID3 is not satisfactory enough, Maria decides to execute again the ID3 but

with different parameters. Now the results are much better and Maria is satisfied with the obtained results. Finally, she saves her work and logs out the system. At this point, the system learns the new experience with its corresponding solution: the ID3 algorithm with the parameters defined by Maria and the CN2 with the default parameters. In figure 2 can be observed some screenshots of the DM assistant interface.
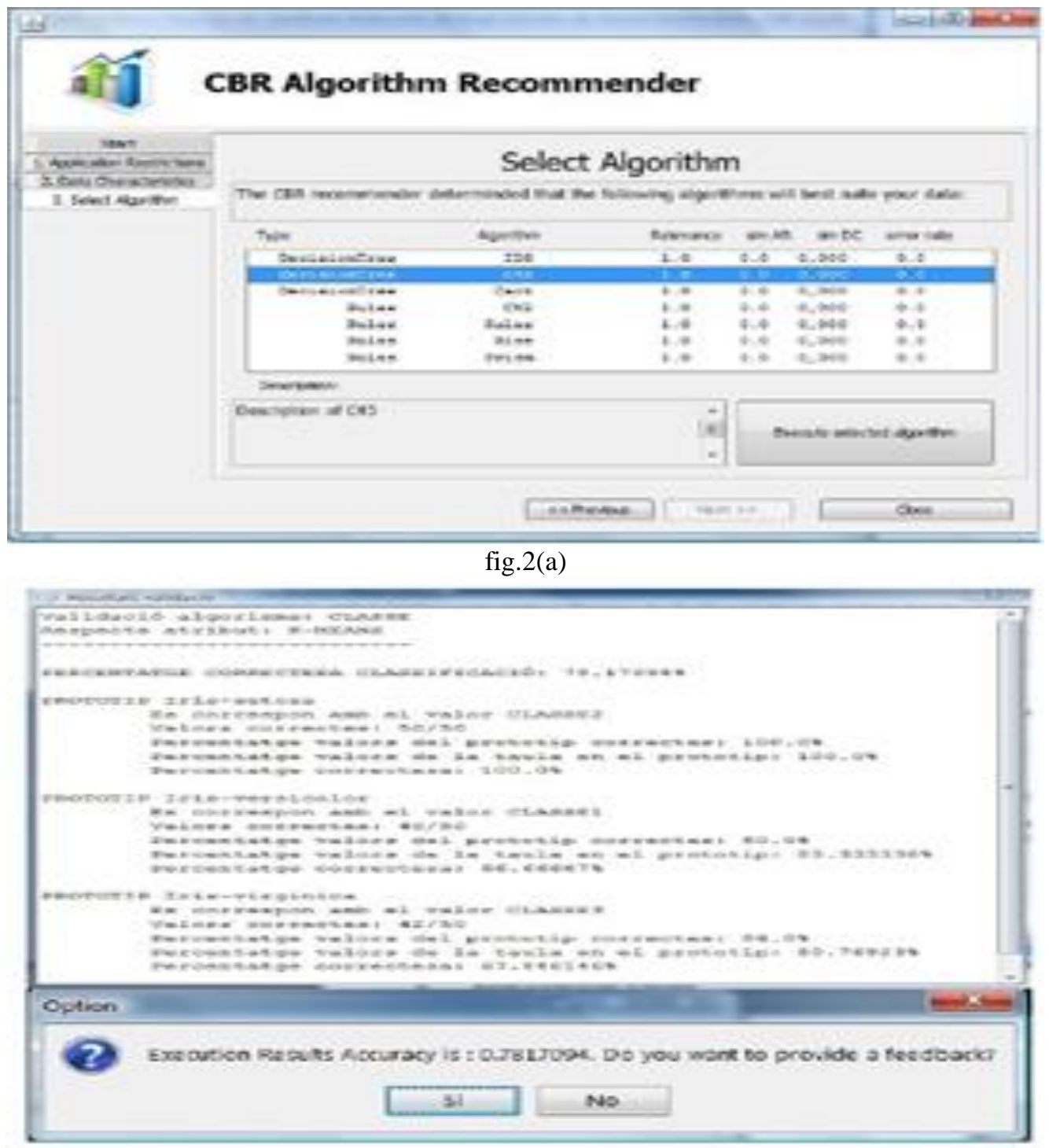

fig.2(b)

Figure 2. (a) screenshot of the DM assistant with an algorithm suggestion; (b) results evaluation results after some algorithm run and, asking to the user for explicit feedback.

\subsection{Evaluation}

A small-scale evaluation was carried out with 5 data miners that knew the original system without the DM assistant integrated. The experiment consisted of each user trying to solve 3 classification problems (P1, $\mathrm{P} 2$, P3), quite similar in terms of dataset characteristics, in different user's sessions and in a sequential manner (i.e. first all users had to solve P1, then P2 and finally P3). Doing it so, all users should be able to reuse the past experiences of others users or/and their own experiences during the small experiment.

After the execution of the experiment the users provided us some feedback about the usefulness of the assistant. In general, the user's opinions were satisfactory, especially in users that were the last ones solving some of the problems, since they could take more advantage of the past experiences of the rest of users. The 
results shown that the assistant is able to refine the case solutions over time, Therefore are gradually better giving support to the users that try to solve problems similar to the ones that have been solved previously.

However, the strong limitation of the system, usual in CBR, is the need of a number of varied past experiences before the assistant provides appropriate support to most users.

\section{Conclusion And Future Work}

Choosing the proper data mining method is one of the most critical and difficult tasks in the KDD process. In this paper, a conceptual map of the most common data mining techniques has been proposed. There is not a unique and consensual classification of data mining methods in the literature. First main decisional criteria used by human experts in real decisions have been identified and the conceptual map is organized based on them. The proposal helps environmental data miners in the conceptual organization and rational understanding of the broad scope of data mining methods; also helps non-expert data miners to improve decisions in real applications. Finally, this provides formal expert knowledge representation to be transmitted to automatic intelligent recommenders, contributing to approach the integral conception of KDD system.

Additionally, an intelligent data mining techniques recommender is being developed based on same decisional criteria, in order to automatically provide recommendations on the best data mining technique. In order to gain flexibility and adaptability to new methods, a pure case-base reasoning approach has been used.

In the future, the deployment of the recommender would be more reliable in a shared environment (e.g. distributed Web system), where multiple data miners could contribute to the enrichment of the knowledge base reducing so the learning/training time of the DM assistant.

\section{References}

[1] Gibert K, Rodríguez-Silva G, Rodríguez-Roda I, 2010: Knowledge Discovery with Clustering based on rules by States: A water treatment application. Environmental Modelling \&Software 25:712-723

[2] Gibert K, J. Spate, M. Sànchez-Marrè, I. Athanasiadis, J. Comas (2008b): Data Mining for Environmental Systems. In Environmental Modeling, Software and Decision Support. pp 205-228.

[3] Pérez-Bonilla A, K. Gibert 2007: Automatic generation of conceptual interpretation of clustering. In Progress in Pattern Recognition, Image analysis and Applications. LNCS-4756:653-663. Springer

[4] Kdnuggets (2006): http://www.kdnuggets.com/polls/2006/data_mining_methods.htm. Data Mining Methods (Apr 2006)

[5] Spate J, K. Gibert, M. Sànchez-Marrè, E. Frank, J. Comas, I. Athanasiadis, R. Letcher 2006. Data Mining as a tool for environmental scientist. In process $1^{\text {st }}$ IEMSs Workshop DM-TEST 2006. 